Acta Crystallographica Section D

Biological

Crystallography

ISSN 0907-4449

\section{Gwyndaf Evans* and Gérard Bricogne}

Global Phasing Ltd, Cambridge CB3 OAX, England

Correspondence e-mail: gwyndaf@globalphasing.com
C) 2003 International Union of Crystallography Printed in Denmark - all rights reserved

\title{
Triiodide derivatization in protein crystallography
}

Methods for producing protein derivatives using cryosoak techniques with triiodide solutions are described. The methods have been tested using six different proteins. SAD/ SIRAS phasing was attempted for each protein using data measured with conventional $\mathrm{Cu} K \alpha \mathrm{X}$-ray equipment and synchrotron radiation. Refinement of all six derivative structures showed that iodine is able to bind as $\mathrm{I}^{-}$(as observed with standard halide soaks) and also as the polyiodide anions $\mathrm{I}_{3}^{-}$and $\mathrm{I}_{5}^{-}$. The various species are able to bind through hydrogen-bond interactions and to more hydrophobic regions of the protein at surface pockets and in intermolecular and intramolecular cavities. On the whole, the derivative agent behaves promiscuously in terms of its binding to proteins and is capable of generating sufficient phasing power from in-house $\mathrm{Cu} K \alpha$ data to permit structure solution by SAD. The results of the phasing experiments and structure refinements are presented.

\section{Introduction}

Recent advances in macromolecular crystallographic methods have created the opportunity to rethink the way in which heavy-atom derivatives are prepared. Traditional methods of heavy-atom derivative preparation by soaking required the use of very low concentrations of heavy-atom agents and soak times of between a few hours and a few weeks. It was imperative that the crystal samples themselves be stable in the presence of the heavy-atom material and reach equilibrium such that diffraction data could be measured at or just below room temperature. A decade ago and earlier the definition of a good derivative was one which produced a small number of highly occupied and well ordered heavy-atom sites and which was isomorphous with the native crystal form. The generation of poor derivatives, where the substructure could not be determined, was often blamed on 'non-specific' binding.

In recent years, the picture has changed very substantially. The ability to freeze crystal samples in preparation for data collection (Hope, 1988; Garman \& Schneider, 1997) has yielded new methods of derivative preparation based on soaking in typically much higher concentrations of heavyatom agents and for much shorter times. Any degradation of the crystal sample can be halted essentially instantaneously by freezing in liquid $\mathrm{N}_{2}$ or in an evaporated $\mathrm{N}_{2}$-gas stream. In cases where such derivatives contain many heavy-atom sites of varying occupancy and order, methods now exist [e.g. SHELXD (Schneider \& Sheldrick, 2002) and SnB (Miller et al., 1994; Smith et al., 1998)] to locate their positions, even if the substructure consists of many tens of atoms. Furthermore,
Received 10 January 2003

Accepted 10 June 2003 
once heavy-atom sites are located, powerful maximumlikelihood methods of heavy-atom refinement and phasing (e.g. SHARP; de La Fortelle \& Bricogne, 1997) are now better able to extract information from derivative data with many weakly bound heavy atoms, even in the presence of nonisomorphism.

The most striking example of how these methods have been used to full advantage is the work of Dauter \& Dauter (1999) and Dauter et al. (2000), who describe the method of short cryosoaking with the halide anions $\mathrm{Br}^{-}$and $\mathrm{I}^{-}$. The authors soak crystal samples in molar solutions of $\mathrm{NaBr}$ or $\mathrm{KI}$ for a few seconds and then freeze the sample in preparation for data collection. They observe halide anions binding with a range of occupancy and temperature factors and show that goodquality phases can still be obtained with MAD and SAD strategies. This work was extended with the use of cation and anion binding by Korolev et al. (2001), who used surfacebound $\mathrm{Rb}$ ions for phasing, by Nagem et al. (2001), who used the compounds $\mathrm{CsCl}, \mathrm{GdCl}_{3}$ and $\mathrm{NaI}$, and by Evans \& Bricogne (2002), who used $\mathrm{NaCl}, \mathrm{CsCl}, \mathrm{NaI}$ and $\mathrm{CsI}$ to generate phase information in a combinatorial MIRAS experiment.

The latter authors also described how triiodide solutions $\left(\mathrm{KI} / \mathrm{I}_{2}\right)$ could be used to produce heavy-atom derivatives capable of generating phase information by SAD using conventional $\mathrm{Cu} K \alpha \mathrm{X}$-ray sources. In this paper, we describe the preparation of triiodide derivatives and summarize the results obtained so far from phasing experiments with inhouse data and more recently with data measured using synchrotron radiation.

\section{Materials and methods}

\subsection{Crystal samples}

Six proteins were used to determine the methods of preparation of triiodide derivatives. Four of these were obtained off-the-shelf: D-xylose isomerase (XI), elastase from porcine pancreas (PPE), hen egg-white lysozyme (HEWL) and apoferritin from horse spleen (APO). Detailed descriptions of crystal sample preparation for these samples can be found in Evans \& Bricogne (2002). Crystals of the two other proteins, antibody SPE7 (ABF; James et al., 2003) and the ear domain of $\gamma$-adaptin (EAR; Kent et al., 2002), were kindly supplied by Leo James (MRC-CPE, Cambridge, England) and Helen Kent (MRC-LMB, Cambridge, England), respectively.

\subsection{Triiodide solutions}

Polyiodide solutions may be prepared by dissolving molecular iodine in solutions of KI. In solution, iodide ions will interact with one or more $\mathrm{I}_{2}$ molecules and form anions $\mathrm{I}_{m}^{n-}$ (Cotton \& Wilkinson, 1988). The most common and stable of these anions is $\mathrm{I}_{3}^{-}$, formed from one $\mathrm{I}^{-}$anion and one $\mathrm{I}_{2}$ molecule. In solution, this anion is usually linear and symmetrical. It may deviate slightly from linearity in crystalline environments and the I-I distance may vary consider-
Table 1

Cryosoak conditions for triiodide derivatization.

The concentration of $\mathrm{KI} / \mathrm{I}_{2}$ is expressed as a $(v / v)$ percentage of the stock solution.

\begin{tabular}{|c|c|c|c|}
\hline Crystal & Cryosoak solution & $\begin{array}{l}\mathrm{KI} / \mathrm{I}_{2} \\
\text { conc. } \\
(\%)\end{array}$ & $\begin{array}{l}\text { Soak } \\
\text { time } \\
\text { (min) }\end{array}$ \\
\hline XI & $\begin{array}{l}1.8 M\left(\mathrm{NH}_{4}\right)_{2} \mathrm{SO}_{4}, 10 \mathrm{~m} M \text { Tris-HCl pH 7.5, } \\
1.86 \mathrm{M}_{\mathrm{L}-\mathrm{x} y l o s e}\end{array}$ & 1 & 8 \\
\hline $\mathrm{ABF}$ & $\begin{array}{l}0.5 M\left(\mathrm{NH}_{4}\right)_{2} \mathrm{SO}_{4}, 0.1 M \mathrm{Na} \text { citrate } \mathrm{pH} 7.5 \\
\quad \mathrm{LiSO}_{4}, 10 \% \text { glycerol }\end{array}$ & 2 & 5 \\
\hline HEWL & $\begin{array}{l}2.5 M \mathrm{NaCl}, 50 \mathrm{~m} M \mathrm{CH}_{3} \mathrm{COONa} \mathrm{pH} 4.7 \\
25 \% \text { ethylene glycol }\end{array}$ & 8 & 10 \\
\hline PPE & 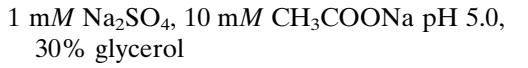 & 10 & 5 \\
\hline APO & $\begin{array}{l}1.25 \mathrm{M}\left(\mathrm{NH}_{4}\right)_{2} \mathrm{SO}_{4}, 10 \mathrm{mM} \mathrm{CdSO}_{4} \\
25 \% \text { ethylene glycol }\end{array}$ & 2 & 5 \\
\hline EAR & $\begin{array}{l}0.9 M \mathrm{Na} / \mathrm{K} \text { tartrate, } 50 \mathrm{~m} M \text { HEPES } \mathrm{pH} \text { 7.4, } \\
20 \% \text { glycerol }\end{array}$ & 5 & 1 \\
\hline
\end{tabular}

ably. Indeed, I-I distances of up to $3.5 \AA$ are still indicative of bond formation.

\subsection{Preparation of derivatives}

For HEWL, APO, XI, PPE and EAR, triiodide derivatives were prepared by soaking the crystals in cryosolutions containing a low concentration of $\mathrm{KI} / \mathrm{I}_{2}$. A stock solution of $\mathrm{KI} / \mathrm{I}_{2}$ was prepared by dissolving $1 \mathrm{~g} \mathrm{KI}$ in $4 \mathrm{ml}$ of water and then adding $0.54 \mathrm{~g} \mathrm{I}_{2}$, giving final concentrations of $0.47 \mathrm{M} \mathrm{I}_{2}$ and $0.67 M \mathrm{KI}$. This solution was then diluted 10-100 times in the protein mother liquor or cryosoak solution such that iodine was present in $\sim 5-50 \mathrm{~m} M$ quantities. The cryosoak drop size was typically $15-20 \mu$ l. Conditions for soaking are given in Table 1. It is important to note that these solutions should be prepared fresh before use, as their composition can change in response to exposure to visible light.

For $\mathrm{ABF}$, the $\mathrm{KI} / \mathrm{I}_{2}$ derivative was prepared by making a $10 \%(v / v)$ solution of $\mathrm{KI} / \mathrm{I}_{2}$ in glycerol and then adding $0.8 \mu \mathrm{l}$ of this directly to a $4 \mu \mathrm{l}$ drop containing crystals. The drop was left until a significant change from colourless crystals to dark yellow/brown had been observed. A crystal from the drop was then mounted directly in the cryostream.

In most cases, the uptake of iodine from the drop into the crystals was clearly visible as a darkening of the crystals from clear to pale yellow and through to brown. The drop itself generally lightened in colour. This was a strong indicator of binding of iodine, in whatever form, to the protein molecules in the crystal as opposed to mere penetration by the iodine into the crystal's solvent channels.

The crystals were soaked for as long as possible in as high a concentration of $\mathrm{KI} / \mathrm{I}_{2}$ as possible. During soaking the crystals were constantly watched and as soon as any degradation of the samples was spotted they were removed with a cryoloop and frozen immediately.

In MASC experiments described by Ramin et al. (1999), in which $3.5 \mathrm{M}\left(\mathrm{NH}_{4}\right)_{2} \mathrm{SeO}_{4}$ was used to soak crystals of $\mathrm{P} 64 \mathrm{~K}$, very high levels of background generated by X-ray fluorescence near the Se $K$ edge seriously degraded the quality of the diffraction data. In the present work, the heavy-atom 
Table 2

Data-integration and scaling statistics for XI, ABF, APO and HEWL derivative data sets and PPE native and derivative sets.

Also given are observed and calculated (retrospectively using refined I occupancies) mean anomalous difference ratios $(\langle|\Delta F|\rangle /\langle F\rangle)$ for the $\mathrm{KI} / \mathrm{I}_{2}$-soaked data sets. Values in parentheses refer to the highest resolution shell.

\begin{tabular}{|c|c|c|c|c|c|c|c|}
\hline \multirow[b]{2}{*}{ Data set } & \multirow[b]{2}{*}{ Resolution $(\AA)$} & \multirow[b]{2}{*}{ Multiplicity } & \multirow[b]{2}{*}{ Completeness (\%) } & \multirow[b]{2}{*}{$R_{\text {merge }}$} & \multirow[b]{2}{*}{$I / \sigma(I)$} & \multicolumn{2}{|l|}{$\langle|\Delta F|\rangle /\langle F\rangle$} \\
\hline & & & & & & Observed & Calculated \\
\hline \multicolumn{8}{|c|}{ Rotating-anode $\mathrm{Cu} K \alpha, 1.5418 \AA\left[f^{\prime \prime}(I)=6.84 \mathrm{e}\right]$} \\
\hline $\mathrm{ABF}\left(\mathrm{KI} / \mathrm{I}_{2}\right)$ & $27.32-1.86(1.96-1.86)$ & $16.9(12.3)$ & $99.7(98.3)$ & $4.2(9.7)$ & $12.8(6.7)$ & 0.041 & 0.042 \\
\hline PPE (native) & $29.88-1.63(1.74-1.63)$ & $3.0(1.2)$ & $77.7(17.7)$ & $5.7(20.9)$ & $10.3(1.9)$ & $\mathrm{n} / \mathrm{a}$ & \\
\hline $\mathrm{PPE}\left(\mathrm{KI} / \mathrm{I}_{2}\right)$ & $26.68-1.85(1.95-1.85)$ & $12.5(7.4)$ & $99.2(95.0)$ & $10.5(34.4)$ & $6.6(2.1)$ & 0.106 & 0.073 \\
\hline $\operatorname{APO}\left(\mathrm{KI} / \mathrm{I}_{2}\right)$ & $22.14-2.01(2.12-2.01)$ & $9.1(6.2)$ & $98.9(93.0)$ & $7.3(32.1)$ & $10.1(2.4)$ & 0.093 & 0.049 \\
\hline $\operatorname{EAR}\left(\mathrm{KI} / \mathrm{I}_{2}\right)$ & $17.99-1.78(1.88-1.78)$ & $5.4(5.1)$ & $99.8(99.8)$ & $3.5(19.5)$ & $13.5(3.7)$ & 0.042 & 0.044 \\
\hline
\end{tabular}

Table 3

Summary of results of phase determination, solvent flattening and automatic model building.

Shown are the number of heavy-atom sites initially found and, in parentheses, the number finally used in phase determination. Also given are the optimal solvent contents determined by autoSHARP (SC), phasing powers (PP), phase correlation coefficients (PCC) and the mean absolute phase difference $(\triangle \varphi)$ relative to the refined derivative structures. The $A R P / w A R P$ results are given as $n / m(c)$ : the number of correctly built residues $n$ out of a total of $m$ that $A R P / w A R P$ was able to auto-build and dock into the amino-acid sequence using $c$ separate chains if breaks occurred. Also given are the r.m.s. deviations between coordinates built and refined by $A R P / w A R P$ and those refined with $B U S T E R / T N T$ for $\mathrm{C}^{\alpha}$ atoms only and, in parentheses, for all atoms. The high value for the latter r.m.s. differences are a consequence of small numbers of incorrectly positioned side chains.

\begin{tabular}{|c|c|c|c|c|c|c|c|c|c|c|c|}
\hline \multirow[b]{2}{*}{ ID } & \multirow[b]{2}{*}{ No. sites } & \multirow[b]{2}{*}{ SC (\%) } & \multirow{2}{*}{$\frac{\text { PP centric }}{\text { Iso }}$} & \multicolumn{2}{|c|}{ PP acentric } & \multicolumn{2}{|c|}{$S H A R P$} & \multicolumn{2}{|c|}{ SOLOMON } & \multicolumn{2}{|l|}{$A R P / w A R P$} \\
\hline & & & & Iso & Ano & PCC & $\Delta \varphi\left(^{\circ}\right)$ & PCC & $\Delta \varphi\left(^{\circ}\right)$ & No. residues & R.m.s.d. ( $\left({ }^{\prime}\right)$ \\
\hline XI & $10(36)$ & 51 & - & - & 1.69 & 0.56 & 42.6 & 0.91 & 15.5 & $376 / 388(3)$ & $0.06(0.76)$ \\
\hline PPE & $2(7)$ & 51 & 0.99 & 1.29 & 1.23 & 0.50 & 45.3 & 0.62 & 41.9 & $219 / 240(6)$ & $0.18(0.87)$ \\
\hline EAR & $6(8)$ & 47 & - & - & 1.49 & 0.49 & 44.7 & 0.85 & 20.9 & $119 / 120(1)$ & $0.08(0.99)$ \\
\hline
\end{tabular}

concentration is lower by an order of magnitude and the fluorescence yield of iodine compared with selenium is also an order of magnitude lower, so that we expected the X-ray fluorescence background to be negligible. This was indeed the case and no back-soaking of the crystals in an iodine-free solution was attempted.

\subsection{Data collection and analysis}

Diffraction data from the EAR crystals were recorded on the BM14-UK CRG beamline at the ESRF. All other data were recorded using rotating-anode $\mathrm{Cu} K \alpha$ radiation.

Diffraction data were integrated using either MOSFLM (Leslie, 1992) or $d^{*} T R E K$ (Pflugrath, 1999). Conversion from $d^{*} T R E K$ integrated reflection files to multi-record MTZ file format was made using DTREK2SCALA. ${ }^{1}$ Scaling and merging was performed with SCALA (Evans, 1997), which applied a empirical spherical harmonic absorption correction to the data. Heavy-atom positions were found either with RANTAN (Yao, 1981) within the autoSHARP procedure (Vonrhein \& Bricogne, 2003) or by using SHELXD (Schneider \& Sheldrick, 2002).

Heavy-atom refinement and phasing was performed using $S H A R P$, either within autoSHARP or through its graphical user interface SUSHI. The resulting experimental electron-

\footnotetext{
${ }^{1}$ Developed by GE from the $C C P 4$ program $A B S U R D$ to convert $d^{*} T R E K$ reflection files and complete geometric information into multi-record MTZ format. To be included in $C C P 4$ release 5.0.
}

density maps were solvent flattened using SOLOMON (Leslie, 1987; Abrahams \& Leslie, 1996) and DM (Cowtan \& Main, 1998), using the autoSHARP protocols.

Refinement of all six derivative structures was performed with BUSTER/TNT (Bricogne, 1997; Tronrud et al., 1987) using starting models taken from the Protein Data Bank.

\section{Results}

The results of data integration and scaling are summarized in Table 2. Care was taken during data collection to measure complete data for all the derivatives. Unfortunately, this was not possible for the native PPE data set owing to restrictions on access to apparatus. The rotating-anode data sets were measured with relatively high multiplicity. The final column of Table 2 shows the observed and calculated mean anomalous difference ratios $\langle|\Delta F|\rangle /\langle F\rangle$. The calculated values were determined using the refined occupancies for all anomalous scatterers identified in the refined structures of the derivatives. The values correlate well over the six data sets apart from the APO case, where the observed ratios are significantly higher than those expected. This is probably owing to the presence of residual errors after scaling and/or poor overall data quality.

\subsection{Phasing}

Structure solution was first attempted for each SAD data set by fully automated means using autoSHARP. This 


\section{Table 4}

Refinement statistics from $B U S T E R / T N T$ for the $\mathrm{KI} / \mathrm{I}_{2}$-soaked structures.

The number of I atoms included in the refinement are given along with, in parentheses, the number of I atoms which form polyhalides. Ramachandran plot statistics were calculated using PROCHECK (Laskowski et al., 1993).

\begin{tabular}{|c|c|c|c|c|c|c|}
\hline & XI & $\mathrm{ABF}$ & PPE & APO & HEWL & EAR \\
\hline Protein atoms & 3050 & 1743 & 1836 & 1398 & 1001 & 822 \\
\hline I atoms & $29(19)$ & $13(6)$ & $23(20)$ & $9(6)$ & $5(2)$ & $8(8)$ \\
\hline Solvent waters & 347 & 139 & 180 & 145 & 99 & 82 \\
\hline Resolution range $(\AA)$ & $32.05-1.55$ & $27.34-1.86$ & $26.68-1.85$ & $22.14-2.01$ & $15.09-1.77$ & $17.99-1.78$ \\
\hline No. reflections & 66821 & 18574 & 19184 & 17272 & 11832 & 12580 \\
\hline Overall completeness (\%) & 98.6 & 99.7 & 99.2 & 98.9 & 99.9 & 99.8 \\
\hline$R_{\text {work }}$ & 16.4 & 18.5 & 16.9 & 18.0 & 17.2 & 19.9 \\
\hline$R_{\text {free }}(5 \%$ data omitted $)$ & 18.8 & 22.8 & 20.5 & 20.5 & 20.8 & 25.8 \\
\hline \multicolumn{7}{|l|}{ Geometry: r.m.s. deviations in } \\
\hline Bond lengths $(\AA)$ & 0.018 & 0.018 & 0.019 & 0.019 & 0.017 & 0.015 \\
\hline Bond angles $\left({ }^{\circ}\right)$ & 1.619 & 1.386 & 1.663 & 1.283 & 1.353 & 1.440 \\
\hline \multicolumn{7}{|c|}{ Ramachandran plot: \% residues in } \\
\hline Most favoured regions & 92.1 & 91.1 & 85.9 & 94.7 & 86.7 & 89.7 \\
\hline Additional allowed regions & 7.3 & 8.4 & 13.6 & 5.3 & 13.3 & 10.3 \\
\hline Generously allowed regions & 0.6 & 0.0 & 0.5 & 0.0 & 0.0 & 0.0 \\
\hline Disallowed regions & 0.0 & 0.5 & 0.0 & 0.0 & 0.0 & 0.0 \\
\hline
\end{tabular}

inspection of the residual maps resulted in the rejection of five sites and the inclusion of four new sites. A second round of $S H A R P$ refinement with these 11 sites was followed by solventflattening optimization and determination of the hand of the heavy-atom solution. After recycling several times through residual map inspection, rejecting poor and accepting new heavy-atom sites, the total number was brought to 20, including a pair of $\mathrm{S}$ atoms identified as a disulfide. An experimental map produced using these 20 sites was input to SOLOMON. ARP/ $w A R P$ built a model into the solventflattened map consisting of 210 residues in five separate chains.

3.1.3. PPE. By itself, the SAD data set did not generate sufficient phase information to permit structure determina-

succeeded for XI and ABF; however, for EAR it was necessary to use $S H E L X D$ to find the sites, which were then input into autoSHARP. For PPE, a native data set was required to provide sufficient phase information to allow structure solution.

For HEWL and APO heavy-atom detection failed, as did heavy-atom refinement and phasing using the known heavyatom positions obtained from anomalous difference Fourier analysis. The reason for this failure was a combination of a high proportion of sites lying on special positions with respect to the crystal symmetry and a poor anomalous signal-to-noise ratio.

The successful cases had crystal solvent-content values of between 46 and 51\%. The two unsuccessful cases, HEWL and APO, had the lowest (37\%) and highest (62\%) solventcontent values, respectively. This suggests that the solvent content of the crystals was not the determining factor in the phasing of these structures. There was also no apparent correlation between the rate of penetration of triiodide into the crystals and their values of solvent content.

The results of the structure-determination efforts are summarized in Table 3 and are detailed below.

3.1.1. XI. Heavy-atom detection using data to $2.0 \AA$ resolution with RANTAN initially determined ten heavy-atom positions. After refinement in $S H A R P$ and automatic interpretation of residual maps, two sites were rejected and 17 new sites were identified. The autoSHARP protocol correctly identified the hand of the heavy-atom constellation and SOLOMON then produced an interpretable $2.0 \AA$ map.

Using these 25 sites, $S H A R P$ was then run with all data to $1.55 \AA$. Residual maps allowed 11 additional sites to be identified and after solvent flattening $A R P / w A R P$ built 376 out of 388 residues of the structure.

3.1.2. ABF. Data between 26.82 and $1.86 \AA$ were used as input to autoSHARP. Initially, RANTAN found 12 heavyatom sites, which were refined with $S H A R P$. Automatic tion. It was necessary therefore to use a native data set of PPE along with data from the $\mathrm{KI} / \mathrm{I}_{2}$-soaked crystal to perform SIRAS phase determination. Even though the quality of the native data was poor, having a low multiplicity and only $77.7 \%$ completeness, it still provided sufficient isomorphous difference signal to allow the sites to be found and the structure to be solved. Native and derivative data extending to 1.63 and $1.85 \AA$, respectively, were input into autoSHARP. RANTAN initially found two heavy-atom sites using the anomalous differences for the derivative data and after an initial SHARP run, three additional sites were added to the heavy-atom model based on residual map peaks. The hand of the heavyatom model was correctly selected on the basis of $E^{2}$ correlation coefficients, but the resulting map was not of sufficient quality to allow automatic building with $A R P / w A R P$. Manual inspection of the residual maps showed evidence of anisotropy in the three strongest iodine sites. Therefore, an additional round of heavy-atom refinement using anisotropic $B$ values was performed, following which two new sites were identified in the isomorphous residual map. Solvent flattening with these phases produced an electron-density map into which $A R P /$ $w A R P$ was able to build $219 / 240$ residues as six polypeptide chains.

3.1.4. EAR. In this case, $R A N T A N$ failed to find a useful solution of heavy-atom positions. However, SHELXD found six sites which were input to $S H A R P$. After cycling three times through residual map inspection and heavy-atom refinement, eight heavy-atom sites were used to produce an experimental map for solvent flattening. ARP/wARP was able to build 119 out of 120 residues into the resulting map.

\subsection{Refinement}

The results of the refinements for all six derivative structures are shown in Table 4. Further details of the refinement 
procedures and results are to be found in Evans \& Bricogne (2002).

Inspection of the six structures showed that iodine introduced as $\mathrm{KI} / \mathrm{I}_{2}$ binds both as iodide anions and as triiodide and pentaiodide anions. Binding of $\mathrm{I}^{-}$anions was via hydrogen bonds to main-chain amide groups of Trp, Tyr, Phe, Leu, Gly, Arg and Asn, to side-chain N atoms of Gln, Lys, Arg and Asn, to hydroxyl groups of Ser and to $\mathrm{N}$ atoms of the Trp indole ring. The polyiodides $\mathrm{I}_{3}^{-}$and $\mathrm{I}_{5}^{-}$bound through hydrogen bonding and through dispersion interactions to hydrophobic regions of the protein molecules. Typical was the stacking of the triiodides against Trp, Pro, Phe and Tyr residues and interaction with the hydrophobic regions of the long sidechain residues Arg, Lys and Asp. Also observed were $\pi$-interactions with His rings. Examples of binding are presented in Fig. 1. Fig. 2 shows a triiodide molecule binding in one of the Xe-binding sites of EAR reported by Kent et al. (2002). This leads us further to the conclusion that triiodide binds comfortably in hydrophobic pockets.

\section{Discussion}

The results demonstrate clearly that derivatization using $\mathrm{KI} / \mathrm{I}_{2}$ solutions can produce sufficient phasing power to allow

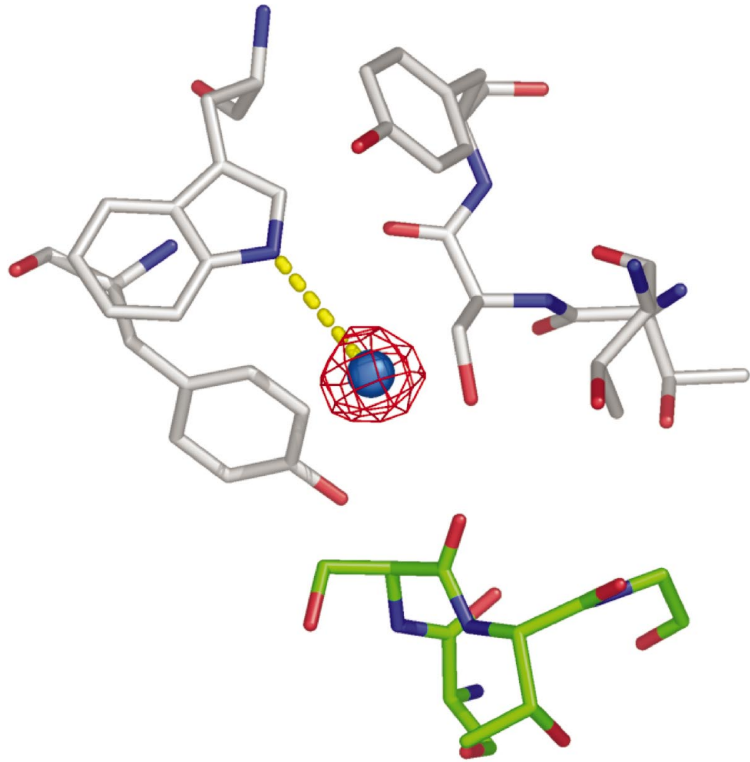

(a)

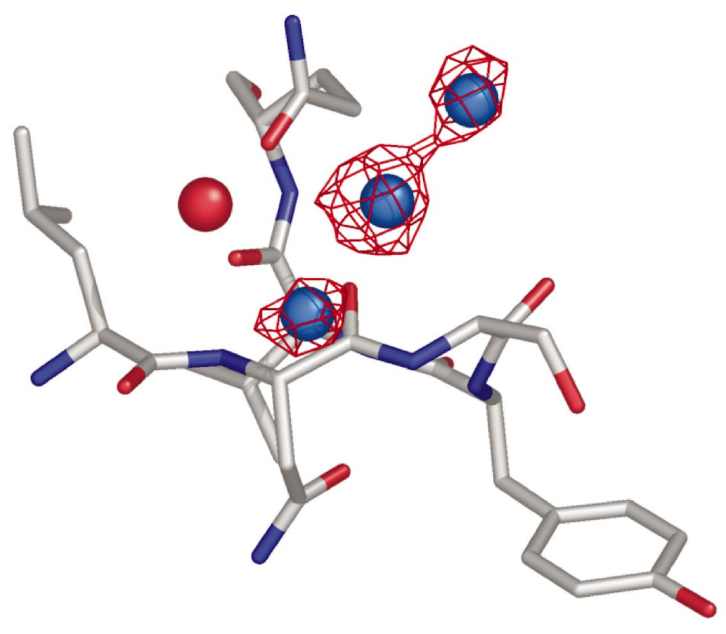

(c)

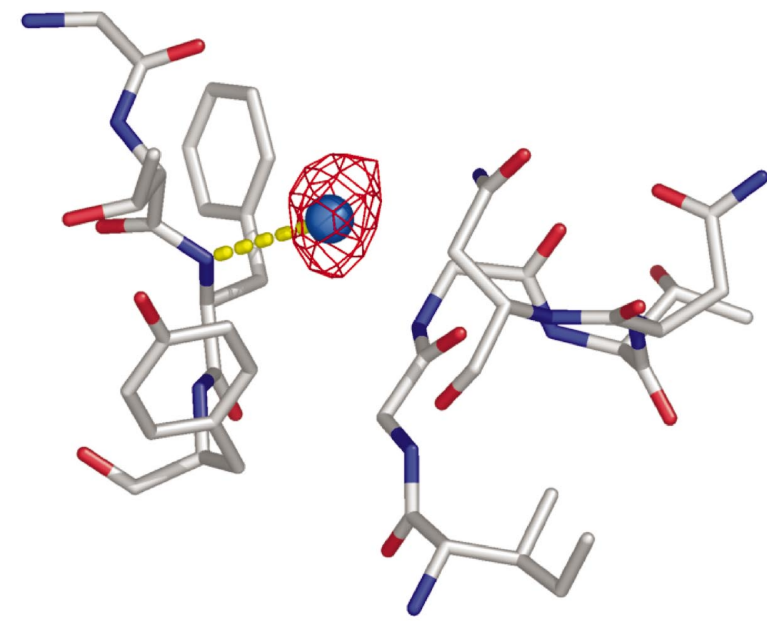

(b)

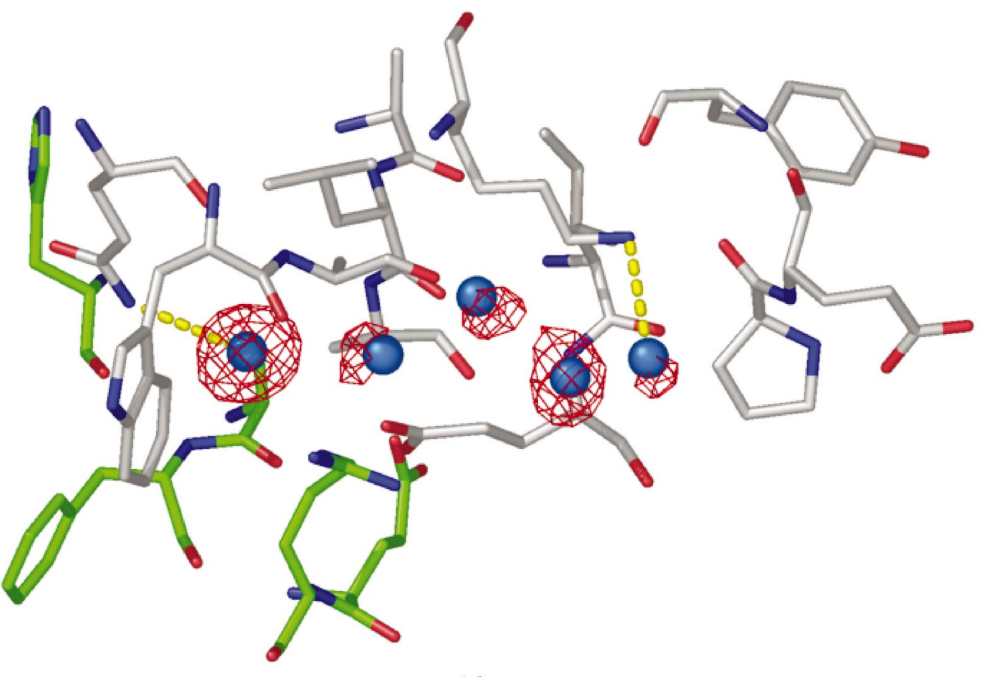

$(d)$

Figure 1

Hydrogen bonds (yellow dashes) in ABF between (a) iodide B71 and TrpH100 NE1 and (b) iodide B91 and the main-chain N atom of PheH105. (c) Triiodide B21-B23 (blue spheres) in a surface pocket of PPE. The triiodide make van der Waals contacts with backbone atoms of Asn115 and Ser116 on one side and the side chain of Gln119 on the other. The close proximity of Wat153 (red sphere), which is $3.06 \AA$ from B21 and $3.34 \AA$ from B 22 , suggests that it binds electrostatically to the triiodide. (d) Example of a pentaiodide molecule (sites B21-B25) binding in a partially solvent-exposed pocket formed between two symmetry-related molecules of XI. The pentaiodide is tethered at each end by hydrogen bonds (yellow dashes) formed with Gln234 NE2 and Lys240 NZ. The central three atoms contact through van der Waals interactions. The symmetry-related protein molecule is shown in green. The anomalous difference Fourier map contoured at $4 \sigma$ is shown in red mesh. 
automatic structure determination. The most striking observation, however, is the dramatic reduction is the amount of data required to solve the EAR structure using synchrotron radiation compared with that required to solve XI, PPE and $\mathrm{ABF}$ using conventional $\mathrm{Cu} K \alpha$ radiation. Even with a reduction in $f^{\prime \prime}$ signal from 6.8 e at $\mathrm{Cu} K \alpha$ to 4.8 e at $1.2398 \AA$, an average multiplicity two to three times less was sufficient for successful automated structure determination. This demonstrates that at a synchrotron source high redundancy is not absolutely necessary for phase determination by SAD using triiodide derivatives. This is heartening in that it means sufficient data could be accumulated before the onset of radiation damage, which is so often observed at the more intense beamline at third-generation synchrotron sources (Burmeister, 2000; Ravelli \& McSweeney, 2000).

It was observed that the number of bound I atoms increases roughly proportionally to the accessible surface area of the molecules in the crystal. A value of $8 \pm 2$ I atoms per $1000 \AA^{2}$ was determined for the six proteins tested here. This suggests that even larger structures could be solved using triiodide. Indeed, the apparent promiscuity of the derivative agent would support this prediction.
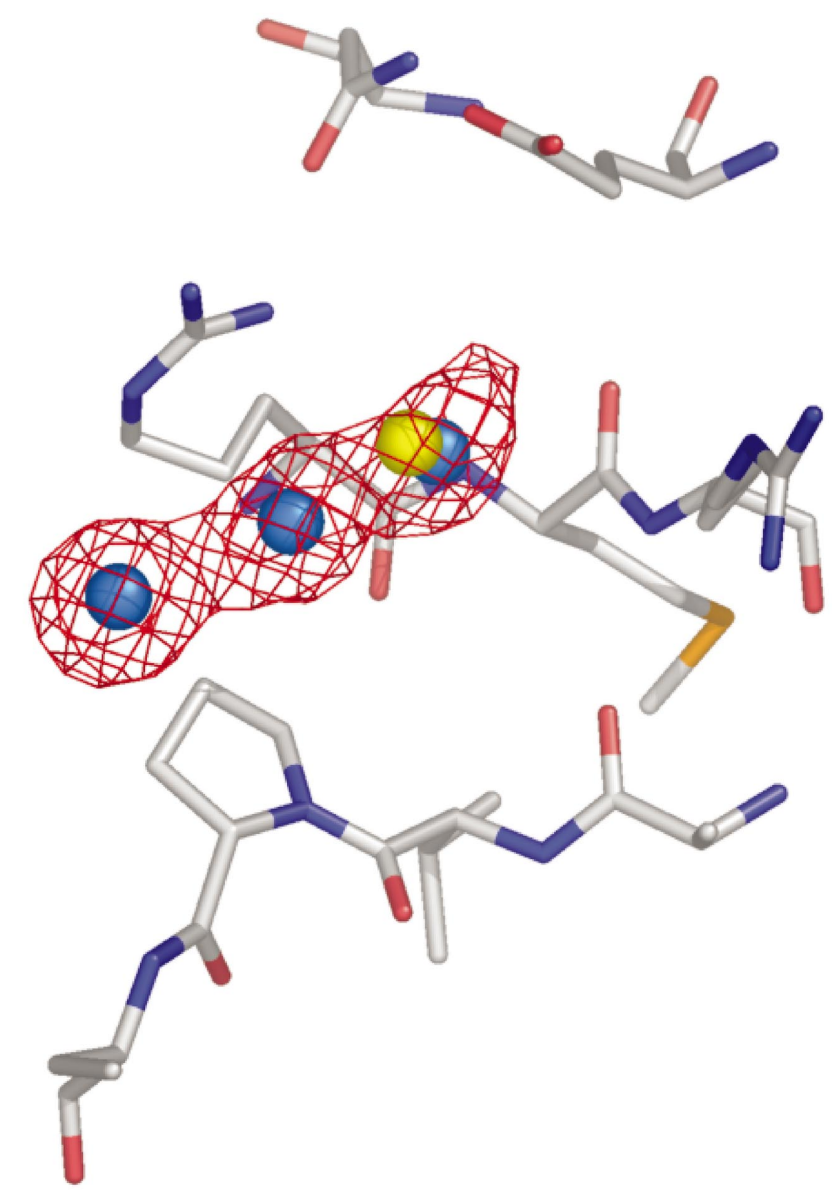

(a)
One interesting feature noticeable in the PPE and EAR derivatives is the ability of triiodide to bind in Xe-binding sites. One disadvantage of $\mathrm{Xe}$ as a derivative is the usually low number of binding sites achieved (Quillin \& Matthews, 2002). Our results suggest that if Xe binds to a protein then there is a good possibility that triiodide will bind in the same site as well as to other sites around the molecule. In the particular case of PPE, seven polyiodide molecules are distributed over the surface of the protein (see Fig. 3).

More recently, it has been observed that $\mathrm{KI} / \mathrm{I}_{2}$ solutions are incompatible with crystallization solutions containing PEG. A phase separation is observed on addition of $\mathrm{KI} / \mathrm{I}_{2}$. This is unfortunate since PEG is widely used in crystallization. Further studies will hopefully reveal a solution to this problem or at least generate alternative ways of introducing triiodide into PEG-based solutions.

\section{Conclusions}

We have demonstrated that solutions of $\mathrm{KI} / \mathrm{I}_{2}$ may be used to generate macromolecular crystal derivatives suitable for phasing by SAD and SIRAS using both conventional $\mathrm{Cu} K \alpha$ and synchrotron radiation.

Iodine introduced to protein crystals in this form is able to bind both through hydrogen bonds as $\mathrm{I}^{-}$and to hydrophobic regions of protein molecules as the polyiodides $\mathrm{I}_{3}^{-}$and $\mathrm{I}_{5}^{-}$. The binding sites are situated in surface pockets and in inter- and intramolecular cavities. The I atoms appear to interact with all accessible amide groups and hydrophobic pockets.

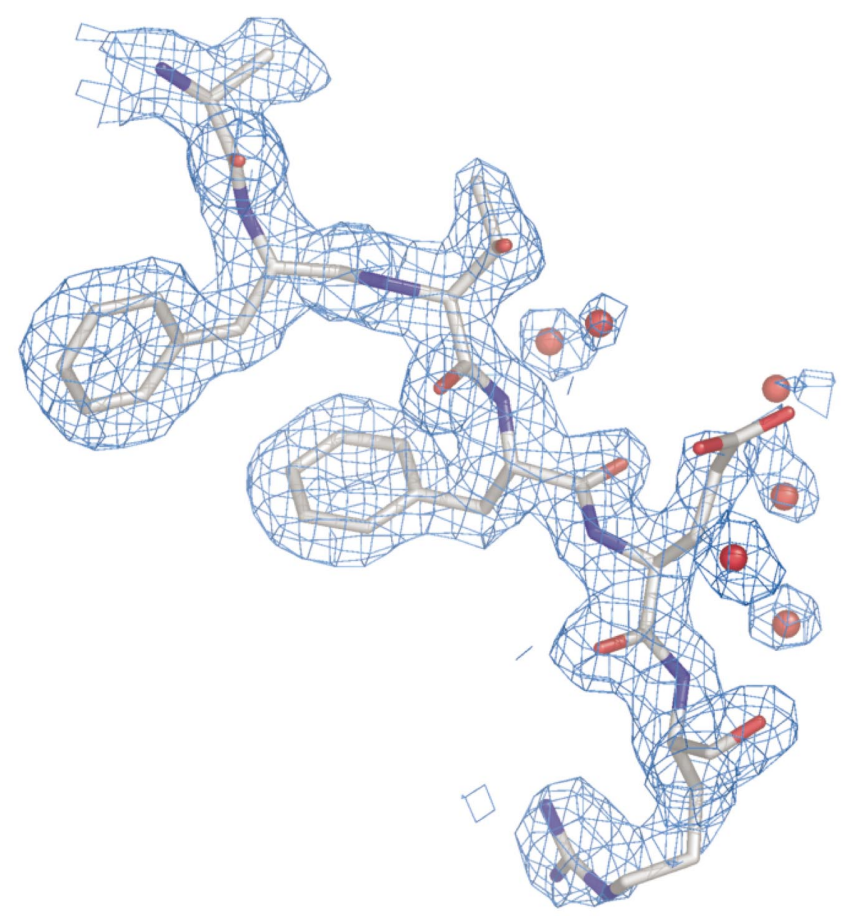

(b)

Figure 2

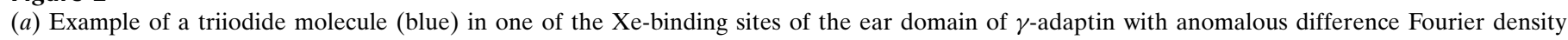

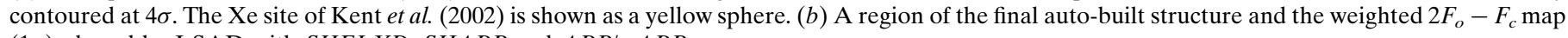

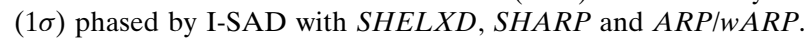




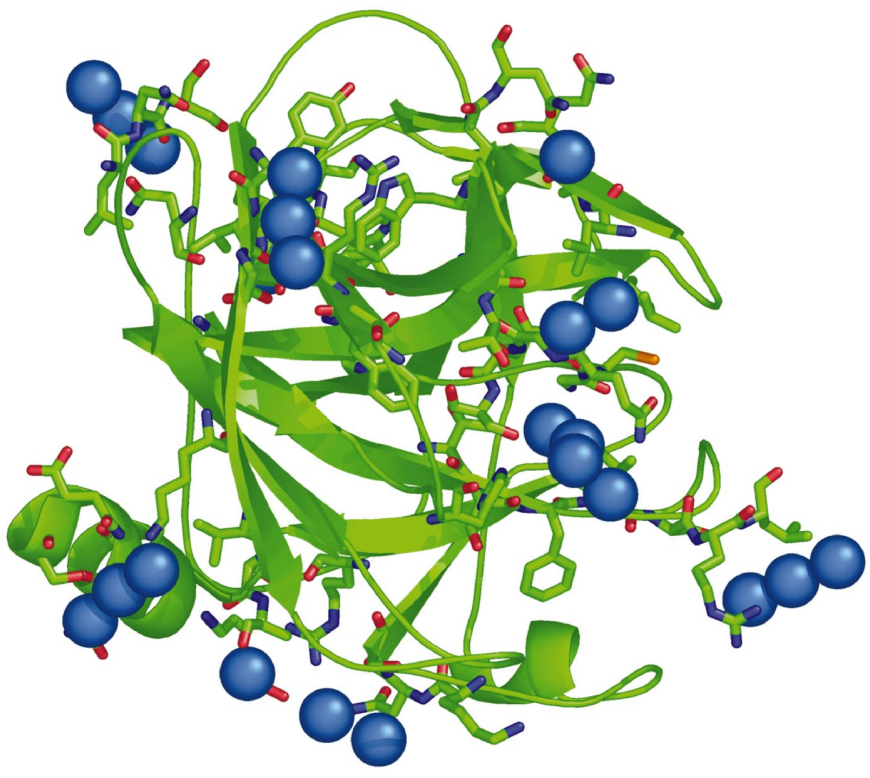

Figure 3

Cartoon representation of the elastase molecule showing seven bound iodides and polyiodides as blue spheres.

The promiscuity of this heavy-atom agent and the ease and speed with which derivatives can be prepared make it a promising candidate for use in high-throughput structuredetermination efforts.

We would like to thank Leo James for providing us with crystals of SPE7, Helen Kent for providing crystals of the $\gamma$-adaptin ear domain, Clemens Vonrhein for help with SHARP and autoSHARP, Pietro Roversi and Eric Blanc for advice on BUSTER and Astex Technology for access to datacollection facilities. This work was partly supported by a Sponsored Research Agreement from Pfizer Central Research to GB (1998-2000) and by European Commission grant No. HPRI-CT-1999-50015 within the EXMAD project.

\section{References}

Abrahams, J. P. \& Leslie, A. G. W. (1996). Acta Cryst. D52, 30-42. Bricogne, G. (1997). Methods Enzymol. 276, 361-423.

Burmeister, W. P. (2000). Acta Cryst. D56, 328-341.

Cotton, F. A. \& Wilkinson, G. (1988). Advanced Inorganic Chemistry, 5 th ed. New York: John Wiley \& Sons.

Cowtan, K. \& Main, P. (1998). Acta Cryst. D54, 487-493.

Dauter, Z. \& Dauter, M. (1999). J. Mol. Biol. 289, 93-101.

Dauter, Z., Dauter, M. \& Rajashankar, K. (2000). Acta Cryst. D56, 232-237.

Evans, G. \& Bricogne, G. (2002). Acta Cryst. D58, 976-991.

Evans, P. R. (1997). Proceedings of the CCP4 Study Weekend. Recent Advances in Phasing, edited by K. S. Wilson, G. Davies, A. W. Ashton \& S. Bailey, pp. 97-102. Warrington: Daresbury Laboratory. Garman, E. F. \& Schneider, T. R. (1997). J. Appl. Cryst. 30, 211-237. Hope, H. (1988). Acta Cryst. B44, 22-26.

James, L. C., Roversi, P. \& Tawfik, D. S. (2003). Science, 299, 13621367.

Kent, H. M., McMahon, H. T., Evans, P. R., Benmerah, A. \& Owen, D. J. (2002). Structure, 10, 1139-1148.

Korolev, S., Dementieva, I., Sanishvili, R., Minor, W., Otwinowski, Z. \& Joachimiak, A. (2001). Acta Cryst. D57, 1008-1012.

La Fortelle, E. de \& Bricogne, G. (1997). Methods Enzymol. 276, 472494.

Laskowski, R. A., MacArthur, M. W., Moss, D. S. \& Thornton, J. M. (1993). J. Appl. Cryst. 26, 283-291.

Leslie, A. G. W. (1987). Acta Cryst. A43, 134-136.

Leslie, A. G. W. (1992). Jnt CCP4/ESF-EAMCB Newsl. Protein Crystallogr. 26.

Miller, R., Gallo, S. M., Khalak, H. G. \& Weeks, C. M. (1994). J. Appl. Cryst. 27, 613-621.

Nagem, R. A. P., Dauter, Z. \& Polikarpov, I. (2001). Acta Cryst. D57, 996-1002.

Pflugrath, J. W. (1999). Acta Cryst. D55, 1718-1725.

Quillin, M. L. \& Matthews, B. W. (2002). Acta Cryst. D58, 97-103.

Ramin, M., Shepard, W., Fourme, R. \& Kahn, R. (1999). Acta Cryst. D55, 157-167.

Ravelli, R. B. G. \& McSweeney, S. M. (2000). Structure, 8, 315-328. Schneider, T. \& Sheldrick, G. (2002). Acta Cryst. D58, 1772-1779.

Smith, G. D., Nagar, B., Rini, J. M., Hauptman, H. A. \& Blessing, R. H. (1998). Acta Cryst. D54, 799-804.

Tronrud, D. E., Eyck, L. F. T. \& Matthews, B. W. (1987). Acta Cryst. A43, 489-501.

Vonrhein, C. \& Bricogne, G. (2003). In preparation.

Yao, J.-X. (1981). Acta Cryst. A37, 645-649. 